\title{
Prevalence of overweight and obesity among 7-9-year-old children in Aveiro, Portugal: comparison between IOTF and CDC references
}

\author{
Carla Pedrosa ${ }^{1,2, *}$, Flora Correia ${ }^{1}$, Dulce Seabra $^{3}$, Bruno MPM Oliveira ${ }^{1}$, \\ Carlos Simões-Pereira ${ }^{2}$ and Maria D Vaz-de-Almeida ${ }^{1}$ \\ ${ }^{1}$ Faculty of Nutrition and Food Sciences of University of Porto, Rua Dr Roberto Frias, 4200-465 Porto, Portugal: \\ ${ }^{2}$ Department of Endocrinology, Diabetes and Nutrition, Hospital Infante D. Pedro EPE, Avenida Dr Artur Ravara, \\ Aveiro, Portugal: ${ }^{3}$ Health Centre of Aveiro, Praça Rainha D. Leonor, Aveiro, Portugal
}

Submitted 26 March 2009: Accepted 8 September 2009: First published online 13 0ctober 2009

\begin{abstract}
Objective: To estimate the prevalence of overweight and obesity in schoolchildren from Aveiro, Portugal, according to two criteria: the International Obesity Task Force (IOTF) and the US Centers for Disease Control and Prevention (CDC) cut-offs.

Design: Weight, height and waist circumference were measured. Using the BMI, gender- and age-specific prevalences of overweight and obesity were determined according to the IOTF cut-offs extrapolated from an adult BMI of 25 and $30 \mathrm{~kg} / \mathrm{m}^{2}$ and the CDC cut-off values of 85th and 95th BMI percentile.

Setting: Aveiro, Portugal.

Subjects: A random representative sample of 905 children ( 457 boys; 448 girls) aged 7-9 years.

Results: The prevalence of excess weight (overweight and obesity) was lowest according to IOTF cut-offs compared to CDC (28.1\% v.31·2\%), especially obesity $(8.1 \% v .14 \cdot 0 \%)$. However, the CDC and IOTF criteria have a strong agreement (Cohen's $k=0.755 ; P<0.001$ ). There were significant differences in excess weight between boys and girls according to the CDC (26.9\% v. 35.7\%; $P=0 \cdot 003)$. Obese children are younger and the majority present abdominal obesity.

Conclusions: The present study shows a high prevalence of excess weight in Aveiro children, similar to other Portuguese regions and among the highest in Europe, especially in the female gender. The IOTF cut-off values give a lower prevalence of excess weight, namely obesity.
\end{abstract}

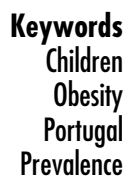

The prevalence of childhood obesity is increasing at a dramatic rate, especially in economically developed countries $^{(1-3)}$. According to international definitions, $10 \%$ of the world's school-aged children is estimated to be overweight, a quarter of which is obese ${ }^{(2,4)}$. Recent data indicate that in Europe, $31.8 \%$ of schoolchildren were estimated to be overweight, including $7 \cdot 9 \%$ estimated to be obese ${ }^{(1,3)}$, with a higher prevalence of overweight youth in Southern Europe ${ }^{(5,6)}$. The significant linear trends in the annual increments indicate that the rate of change of prevalence is itself increasing. The predicted prevalence for 2010 will be $38 \cdot 2 \%$ for overweight, of which $10 \%$ reports to obesity ${ }^{(1,3)}$.

In Portugal, weight and height are not assessed regularly at the national level. In a recent review, fifteen individualised studies reported overweight and obesity, in Portugal, in children and adolescents aged $2-15$ years $^{(7)}$. In a sample of 5511 children (7-9-year-old), a prevalence of overweight between $19 \cdot 1 \%$ and $21 \cdot 4 \%$ and of obesity between $10 \cdot 2 \%$ and $12 \cdot 3 \%$ was reported, respectively, in boys and girls ${ }^{(8)}$.

Obesity has important consequences as it increases the risk of other chronic diseases, including dyslipidaemia, hypertension, CVD, insulin resistance and diabetes. Moreover, obesity influences the physical, social and psychological development of children ${ }^{(9)}$. Approximately one-third of overweight children and one-half of overweight adolescents remain obese as adults ${ }^{(3,10)}$. Therefore, prevention of obesity in these age groups should be a public health priority.

There is no widely agreed standard for classifying overweight and obesity in paediatric age, however the use of a universal classification may facilitate international comparisons $^{(2,11)}$. The BMI is the most practical and widely used index to identify overweight and obese children and adolescents for survey purposes ${ }^{(12)}$. The 
International Obesity Task Force (IOTF) recommends the use of BMI cut-off points of Cole et al. ${ }^{(13)}$. These were derived from the BMI cut-off points of 25 and $30 \mathrm{~kg} / \mathrm{m}^{2}$ at an age of 18 years and by extrapolating these along the equivalent BMI-for-age centile lines in a multinational data set of children aged 6-18 years ${ }^{(13)}$. The US Centers for Disease Control and Prevention (CDC) recommends the sex- and age-specific 85th and 95th BMI percentile as the cut-offs to define overweight and obesity in children and adolescents ${ }^{(14)}$. Both methods are routinely employed to assess childhood obesity ${ }^{(15)}$, nevertheless previous research $^{(16,17)}$ showed that they yield different prevalences for overweight and obesity.

The present study has been carried out in order to estimate the prevalence of overweight and obesity in 7-9year-old children from Aveiro and to compare the IOTF and CDC sex-specific BMI-for-age reference values.

\section{Subjects and methods}

The study was carried out in public primary schools of Aveiro in a sample of 7-9-year-old children. This age group was chosen for physiological reasons, as it precedes puberty ${ }^{(18)}$. The study protocol was approved by each group of schools (forty public primary schools scattered within seven groups of schools).

Aveiro is the fifth most populous district in Portugal. Its gross domestic product (GDP) per capita represents 90\% of the national average, with low levels of poverty ${ }^{(19)}$.

\section{Participants}

The number of children enrolled at 2 nd and 3rd grade (corresponding to children aged between 7 and 9 years) was identified ( $n$ 1799) at the beginning of 2005/2006 academic year. A random sample of schools was extracted with the restriction of having at least one school from each group of schools. The probability of selection of one school was proportional to the number of students in the 2nd and 3rd grade. From a total of forty, thirteen schools were selected (see Appendix). A representative sample of 980 children was obtained, corresponding to all students in the 2nd and 3 rd grade of the sampled schools. The survey was carried out from January to March 2006 and 931 children were evaluated, of which $98.8 \%$ were Caucasian. Twenty-six children were excluded (twenty-five were older than 9 years; one suffered from Down syndrome). Therefore, our sample consists of 905 children ( $92 \cdot 4 \%$ of the initial sample).

\section{Anthropometric measures}

In each school, two trained staff (one nutritionist and one nurse) measured height, weight and waist circumference according to standardised procedures ${ }^{(20)}$. Weight was measured to the nearest $0 \cdot 1 \mathrm{~kg}$ using an electronic column scale (SECA-780; Seca Ltd, Hamburg, Germany); height was measured to the nearest $0 \cdot 1 \mathrm{~cm}$ using a stadiometer
(SECA-220, Seca Ltd), with the head in the Frankfort horizontal plane; waist circumference was measured at the mid-point between the iliac crest and the lower edge of the ribs at the end of a normal expiration. Age was calculated in decimal units based on the date of the survey relative to birth date. Overweight and obesity were defined using the IOTF cut-offs proposed by Cole et $a l^{(13)}$ derived from BMI cut-points of 25 and $30 \mathrm{~kg} / \mathrm{m}^{2}$ and the CDC sex- and age-specific 85th and 95th BMI percentile ${ }^{(14)}$. The term overweight in the present study does not include obese children. Abdominal obesity was defined using the sex and age-specific 95th waist circumference percentile by McCarthy et al. ${ }^{(21)}$.

\section{Statistical analysis}

Statistical analysis was performed using the Statistical Package for the Social Sciences statistical software package version $14 \cdot 0$ (SPSS Inc., Chicago, IL, USA). Descriptive statistics were executed by computing the mean and standard deviation for continuous variables, or frequencies for ordinal and nominal variables. Pearson and Spearman correlation coefficients were computed to evaluate the degree of association between variables. Independent samples $t$ test and ANOVA were performed on scale variables to evaluate the differences between two or more groups, respectively. The Mann-Whitney $U$ test was used in ordinal variables to evaluate the differences between two groups. To study the independence between nominal categorical variables, the $\chi^{2}$ test was used. Cohen's $k$ was calculated in order to measure the agreement between the IOTF and CDC criteria. The IOTF criteria were computed using the Excel add-on lmsGrowth $^{(22)}$ and CDC criteria were computed using the tables provided by the Centers for Disease Control and Prevention webpage ${ }^{(23)}$.

\section{Results}

The analysis involves 905 children aged 7-9 years, of whom $457(50 \cdot 5 \%)$ were boys and 448 (49.5\%) were girls. The characteristics of the sample are shown in Table 1 . There are no significant differences in age and anthropometric characteristics between boys and girls.

The prevalence of overweight and obesity by gender according to IOTF and CDC is shown in Table 2. The prevalence of excess weight (overweight and obesity) was lowest according to IOTF $(28 \cdot 1 \% v .31 \cdot 2 \%)$, especially in the obese group $(8 \cdot 1 \% v \cdot 14 \cdot 0 \%)$. The IOTF and CDC criteria have a strong agreement (Cohen's $k=0 \cdot 755$, $P<0 \cdot 001$ ), however with higher prevalence of obesity when using the CDC. According to IOTF, the prevalence of excess weight was highest among girls, without significance $(Z=-1 \cdot 794, P=0 \cdot 073)$. Also, according to the CDC criteria, the prevalence of both overweight and obesity was highest among girls $(35 \cdot 7 \% v .26 \cdot 9 \%)$, with significant differences between genders for the CDC criteria 
Table 1 Descriptive characteristics of the 7-9-year-old Portuguese children living in Aveiro, according to gender

\begin{tabular}{|c|c|c|c|c|c|c|}
\hline & \multicolumn{2}{|c|}{ Boys ( $n$ 457) } & \multicolumn{2}{|c|}{ Girls ( $n$ 448) } & \multirow[b]{2}{*}{$95 \% \mathrm{Cl}$} & \multirow[b]{2}{*}{$P$} \\
\hline & Mean & SD & Mean & SD & & \\
\hline Age (years) & $8 \cdot 23$ & 0.70 & $8 \cdot 24$ & 0.64 & $-0.06,0.11$ & 0.588 \\
\hline Weight (kg) & $29 \cdot 86$ & $6 \cdot 07$ & $29 \cdot 90$ & $6 \cdot 78$ & $-0.08,0.90$ & 0.887 \\
\hline Height $(\mathrm{cm})$ & $130 \cdot 01$ & $6 \cdot 21$ & $129 \cdot 38$ & $6 \cdot 40$ & $-1 \cdot 42,0 \cdot 22$ & $0 \cdot 151$ \\
\hline BMI $\left(\mathrm{kg} / \mathrm{m}^{2}\right)$ & $17 \cdot 53$ & $2 \cdot 54$ & $17 \cdot 71$ & $2 \cdot 90$ & $-0.18,0.53$ & 0.344 \\
\hline Z-score BMI & $0 \cdot 33$ & $0 \cdot 70$ & 0.25 & 0.56 & $-0.06,0.19$ & 0.299 \\
\hline Waist circumference $(\mathrm{cm})$ & $59 \cdot 87$ & $6 \cdot 37$ & $59 \cdot 28$ & $7 \cdot 27$ & $-1 \cdot 48,0 \cdot 30$ & 0.195 \\
\hline
\end{tabular}

There are no significant differences between boys and girls in relation to all studied variables.

Table 2 Prevalence of overweight and obesity among 7-9-yearold Aveiro children according to the IOTF and the CDC sex-specific BMI-for-age reference values and gender

\begin{tabular}{|c|c|c|c|c|c|c|}
\hline & \multicolumn{2}{|c|}{ Boys ( $n$ 457) } & \multicolumn{2}{|c|}{ Girls ( $n$ 448) } & \multicolumn{2}{|c|}{ All (n 905) } \\
\hline & $n$ & $\%$ & $n$ & $\%$ & $n$ & $\%$ \\
\hline \multicolumn{7}{|l|}{ IOTF } \\
\hline Overweight & 85 & $18 \cdot 6$ & 96 & $21 \cdot 4$ & 181 & $20 \cdot 0$ \\
\hline $\begin{array}{l}\text { Obese } \\
\text { CDC }\end{array}$ & 30 & $6 \cdot 6$ & 43 & $9 \cdot 6$ & 73 & $8 \cdot 1$ \\
\hline Overweight & 74 & $16 \cdot 2$ & 82 & $18 \cdot 3$ & 156 & $17 \cdot 2$ \\
\hline Obese & 49 & $10 \cdot 7$ & 78 & $17 \cdot 4$ & 127 & $14 \cdot 0$ \\
\hline
\end{tabular}

IOTF, International Obesity Task Force; CDC, US Centers for Disease Control and Prevention.

There were significant differences between genders for the CDC criteria (Mann-Whitney $Z=2.966, P=0.003$ ) but no differences were found for the IOTF (Mann-Whitney $Z=-1 \cdot 794, P=0.073$ ) for the mean rank of the BMI group (normal, overweight and obese).

( $Z=2 \cdot 966, P=0 \cdot 003)$ for the mean rank of the BMI group (normal, overweight and obese).

Obese children are younger $(8.25$ (SD 0.67$)$ years in normal weight $v .8 .08$ ( $\mathrm{SD} 0.63$ ) years in obese according to IOTF, and $8 \cdot 15$ (SD $0 \cdot 64$ ) years in obese according to CDC), although the correlation between age and $Z$-score BMI is very weak $(R=-0 \cdot 084, P<0 \cdot 011)$. There is no statistical difference in age $(P=0.588)$ and $Z$-score BMI $(P=0 \cdot 300)$ according to gender. Abdominal obesity (Table 3 ) is present in $84.9 \%$ and $83.5 \%$ of obese children according to IOTF $(\rho=0 \cdot 608 ; P<0 \cdot 001)$ and CDC criteria $(\rho=0 \cdot 639$; $P<0 \cdot 001)$, respectively. Waist circumference is strongly associated with BMI $(R=0 \cdot 922 ; P<0 \cdot 001)$.

According to the school environment (ten rural schools: 458 children; three urban schools: 447 children), the prevalence of excess weight is similar in both school types (IOTF: $27 \cdot 51 \%$ in rural $v .28 \cdot 57 \%$ in urban; CDC: $33.63 \% v$. $32 \cdot 14 \%)$. There is no statistical difference between gender, age and $Z$-score BMI according to rural or urban environment $(P=0 \cdot 154 ; P=0 \cdot 519 ; P=0 \cdot 185)$ and according to each school $(P=0 \cdot 116 ; P=0 \cdot 188 ; P=0 \cdot 092)$.

\section{Discussion}

Our study presents the first estimates on prevalence of overweight and obesity in children living in Aveiro. These
Table 3 Waist circumference percentile distribution in 7-9-yearold normal weight, overweight and obese children according to the IOTF and the CDC sex-specific BMI-for-age reference values

\begin{tabular}{|c|c|c|c|c|c|c|}
\hline & \multicolumn{6}{|c|}{ Waist circumference percentiles } \\
\hline & \multicolumn{2}{|c|}{$<90$ th } & \multicolumn{2}{|c|}{$\geq 90$ th-95th } & \multicolumn{2}{|c|}{$\geq 95$ th } \\
\hline & $n$ & $\%$ & $n$ & $\%$ & $n$ & $\%$ \\
\hline \multicolumn{7}{|l|}{ IOTF } \\
\hline Normal weight ( $n$ 617) & 499 & $80 \cdot 9$ & 62 & $10 \cdot 0$ & 56 & $9 \cdot 1$ \\
\hline Overweight ( $n$ 181) & 40 & $22 \cdot 1$ & 28 & $15 \cdot 5$ & 113 & $62 \cdot 4$ \\
\hline Obese $(n 73)$ & 11 & $15 \cdot 1$ & 0 & $0 \cdot 0$ & 62 & $84 \cdot 9$ \\
\hline \multicolumn{7}{|l|}{ CDC } \\
\hline Normal weight ( $n$ 613) & 511 & $83 \cdot 4$ & 55 & $9 \cdot 0$ & 47 & $7 \cdot 7$ \\
\hline Overweight ( $n$ 156) & 42 & $26 \cdot 9$ & 35 & $22 \cdot 4$ & 79 & $50 \cdot 6$ \\
\hline Obese $(n 127)$ & 20 & $15 \cdot 7$ & 1 & $0 \cdot 8$ & 106 & 83.5 \\
\hline
\end{tabular}

IOTF, International Obesity Task Force; CDC, US Centers for Disease Control and Prevention.

Spearman correlation between waist circumference percentile and IOTF criteria: $\rho=0.608 \quad(P<0.001)$. Spearman correlation between waist circumference percentile and CDC criteria: $\rho=0.639(P<0.001)$.

data constitute a representative sample of 7-9-year-old schoolchildren, although randomisation was school- and not individually based. We found a high prevalence of excess weight (overweight and obesity) between 28\% and $31 \%$ according to the criteria used (IOTF and CDC, respectively), which is estimated to be among the highest in Europe ${ }^{(1)}$. This prevalence is in accordance with Padez et $a l^{(8)}$, which found $31.5 \%$ of excess weight according to IOTF $(20 \cdot 3 \%$ overweight and $11 \cdot 3 \%$ obesity $v .20 \cdot 0 \%$ overweight and $8 \cdot 1 \%$ obesity in our study).

The prevalence of overweight in our sample is slightly higher when using IOTF reference $(20 \cdot 0 \% v$. 17.2\%). However, a higher prevalence of obesity, almost twofold, was found when using the CDC charts $(8 \cdot 0 \% v \cdot 14 \cdot 0 \%)$. Several authors also have found a higher prevalence of obesity when using the CDC reference. Lobstein et al. showed that $10 \cdot 7 \%$ of boys and $14 \cdot 0 \%$ of girls (6-11-yearold) were obese according to IOTF, while according to CDC criteria obesity increased to $19.9 \%$ in boys and $17.6 \%$ in girls (data from the National Health and Nutrition Examination Survey 2003-2004). In contrast, the IOTF reference yielded a higher percentage of overweight compared to CDC $(21 \%$ and $23.6 \%$ for IOTF $v \cdot 16 \cdot 6 \%$ and $20.4 \%$ for CDC, respectively in boys and girls $)^{(24)}$. In a national sample of 6-12-year-old Swiss children, $3 \cdot 85 \%$ 
and $3.72 \%$ of boys and girls were obese based on IOTF reference, whereas $7 \cdot 63 \%$ and $5.91 \%$ were obese based on $\mathrm{CDC}^{(17)}$. In the Pro Children Study, the authors also found that CDC criteria consistently estimated higher proportions of obesity than IOTF ${ }^{(25)}$. These two definitions differ in data sets, smoothing methods and approaches to set cutoffs ${ }^{(16,26)}$. The IOTF BMI cut-off points derived from a multinational (Brazil, United Kingdom, Hong-Kong, Singapore, the Netherlands and USA) data set of children ${ }^{(13)}$. While the CDC2000 growth charts were created to update the 1977 National Center for Health Statistics charts using data from five cross-sectional child growth surveys, involving US nationally representative samples ${ }^{(14)}$. For 6-12-yearolds, the IOTF cut-offs are generally higher than the CDC ones. For boys, the mean differences between the CDC and IOTF references for overweight and obesity are around 0.5 and $1.5-2.0 \mathrm{~kg} / \mathrm{m}^{2}$, respectively. For girls, the two sets of reference values are similar for overweight, but the IOTF cut-offs are near $1.0 \mathrm{~kg} / \mathrm{m}^{2}$ higher ${ }^{(16)}$. Therefore, the IOTF definition of obesity tends to be more cautious and according to several studies its sensitivity for obesity is poorer when compared to CDC definition ${ }^{(17,27)}$. This can lead to an underestimation of obesity prevalence. Although the IOTF reference has a slightly stronger capacity for predicting obesity and metabolic disorders in young adulthood $^{(15)}$, the CDC criteria, being broader, might have the advantage of identifying more children who will benefit from an early intervention, even at the risk of creating false positives.

Differences in gender are generally small and there is no suggestion that either males or females consistently predominate within particular age groups, or according to particular overweight definitions ${ }^{(28,29)}$. In almost all cases, the gender differences were not statistically significant ${ }^{(28)}$. Nevertheless, in our study we found a higher prevalence of excess weight in girls, statistically significant according to CDC. This might be related to gender differences in physical activity, since boys are generally more active than girls ${ }^{(28)}$. However, physical activity levels were not evaluated in our study.

Researchers should be aware of the differences between IOTF and CDC criteria, since they will yield different prevalence rates. Each one has its advantages and limitations, and should be used taking into account its possible limitations ${ }^{(16)}$.

Despite the small age interval of the sample, the prevalence of obesity was higher in younger children, which could be an indicator of a future increase in the prevalence of obesity. It also shows the importance of an early intervention in order to prevent the increase in weight and its consequences.

In our sample, the majority of obese children present abdominal obesity. According to other authors, waist circumference is a useful measure of the abdominal obesity that is more closely related to cardiovascular risk and metabolic syndrome than overall obesity ${ }^{(30,31)}$.
Therefore, waist circumference measurement might be a simple tool to help identify children at risk.

The school environment might be associated with socioeconomic status of families, but we did not find differences in the prevalence of excess weight between children from schools in rural and in urban areas. In the rural area, schools are smaller and children generally come from families that work mainly in agriculture and factories, with a lower education level. In the urban area, children generally come from families that work mainly in the services industry, normally with higher education level. A limitation of our study is that we did not evaluate the family socio-economic status and the parent's education. In industrialised societies, adults with a low/medium education level and less qualified occupations are at risk of excess weight, and also influence the diet and lifestyle of their families, which leads to a greater risk for their children ${ }^{(32)}$. Lower socio-economic status may also be related to decreased physical activity in children and adolescents ${ }^{\text {(33) }}$.

In summary, the prevalence of excess weight in Portuguese schoolchildren living in Aveiro is high, especially in the female gender, and among the highest in Europe. Our data confirm that the CDC charts give a higher prevalence of obesity, compared to the IOTF. The majority of obese children present abdominal obesity, which can be associated with cardiometabolic risk. It is clear that the obesity epidemic calls for an urgent action, to set up surveillance programmes and preventive strategies in order to contain the increasing number of obese children.

\section{Acknowledgements}

Sources of funding: This work was supported by a SPEDM/ ABBOTT grant. Conflicts of interest: None declared. Authors' contributions: C.P. developed the study protocol, participated in child screening and assessment, data analysis and writing the manuscript. F.C. participated in protocol development, data analysis and contributed to the writing of the manuscript. D.S. provided advice on study design, and participated in child screening and assessment. B.M.P.M.O. completed the random sampling and statistical analysis, and provided advice regarding interpretation and presentation of results. C.S.P. and M.D.V.A. provided advice on study design and contributed to the writing of the manuscript. All authors read and approved the final manuscript. Acknowledgements: We thank the children and the teachers who participated in the study. We also like to thank the nurse Ana Lebreiro for her support in the fieldwork and the nutritionist Isabel Albuquerque for review and comments.

\section{References}

1. Jackson-Leach R \& Lobstein T (2006) Estimated burden of paediatric obesity and co-morbidities in Europe. Part 1. The increase in the prevalence of child obesity in Europe is itself increasing. Int J Pediatr Obes 1, 26-32. 
2. Lobstein T, Baur L \& Uauy R, for the IOTF Childhood Obesity Working Group (2004) Obesity in children and young people: a crisis in public health. Obes Rev 5, Suppl. 1, 4-85.

3. Wang Y \& Lobstein T (2006) Worldwide trends in childhood overweight and obesity. Int J Pediatr Obes 1, 11-25.

4. International Obesity Task Force, European Association for the Study of Obesity (2002) Obesity in Europe - The case for action. http://www.iaso.org/popout.asp?linkto $=\mathrm{http} \%$ 3A//www.iotf.org/media/euobesity.pdf (accessed March 2003).

5. Janssen I, Katzmarzyk PT, Boyce WF et al. \& The Health Behaviour in School-Aged Children Obesity Working Group (2005) Comparison of overweight and obesity prevalence in school-aged youth from 34 countries and their relationships with physical activity and dietary patterns. Obes Rev 6, 123-132.

6. Lobstein T \& Frelut M-L (2003) Prevalence of overweight among children in Europe. Obes Rev 4, 195-200.

7. Moreira P (2007) Overweight and obesity in Portuguese children and adolescents. J Public Health 15, 155-161.

8. Padez C, Fernandes T, Mourão I et al. (2004) Prevalence of overweight and obesity in 7-9-year-old Portuguese children: trends in body mass index from 1970-2002. Am J Hum Biol 16, 670-678.

9. Dietz WH (1998) Health consequences of obesity in youth: childhood predictors of adult disease. Pediatrics 101, 518-525.

10. Singh AS, Mulder C, Twisk JWR et al. (2008) Tracking of childhood overweight into adulthood: a systematic review of the literature. Obes Rev 9, 474-488.

11. Reilly JJ (2002) Assessment of childhood obesity: national reference data or international approach? Obes Res 10, 838-840.

12. Dietz WH \& Robinson TN (1998) Use of the body mass index (BMI) as a measure of overweight in children and adolescents. J Pediatr 132, 191-193.

13. Cole TJ, Bellizzi MC, Flegal KM et al. (2000) Establishing a standard definition for child overweight and obesity worldwide: international survey. BMJ 320, 1240-1243.

14. Kuczmarski RJ, Ogden CL, Grummer-Strawn LM et al. (2000) CDC growth charts: United States. Adv Data 314, 1-28.

15. Janssen I, Katzmarzyk PT, Srinivasan SR et al. (2005) Utility of childhood BMI in the prediction of adulthood disease: comparison of national and international references. Obes Res 13, 1106-1115.

16. Flegal KM, Ogden CL, Wei R et al. (2001) Prevalence of overweight in US children: comparison of US growth charts from the Centers for Disease Control and Prevention with other reference values for body mass index. Am J Clin Nutr 73, 1086-1093.

17. Zimmermann MB, Gübeli C, Püntener C et al. (2004) Detection of overweight and obesity in a national sample of 6-12-y-old Swiss children: accuracy and validity of reference values for body mass index from the US Centers for Disease Control and Prevention and the International Obesity Task Force. Am J Clin Nutr 79, 838-843.

18. Lehingue Y (1999) The European Childhood Obesity Group (ECOG) project: the European collaborative study on the prevalence of obesity in children. Am J Clin Nutr 70, Suppl., 166S-168S.

19. Instituto Nacional de Estatística (editor) (2009) Retrato Territorial de Portugal 2007. Lisboa: INE.

20. World Health Organization (1995) Physical Status: The Use and Interpretation of Anthropometry. Report of a WHO Expert Committee. WHO Technical Report Series no. 854. Geneva: WHO.

21. McCarthy HD, Jarret KV \& Crawley HF (2001) The development of waist circumference percentiles in British children aged 5•0-16・9y. Eur J Clin Nutr 55, 902-907.

22. ImsGrowth 2.64. http://www.healthforallchildren.co.uk/ pro.epl?DO $=$ USERPAGE\&PAGE $=$ lmsdownload $\quad($ accessed November 2008).

23. Centers for Disease Control and Prevention (2000) CDC Growth Charts: United States. Percentile data files with LMS values. zbmiage.txt available from http://www.cdc.gov/ nchs/data/nhanes/growthcharts (accessed January 2004).

24. Lobstein T \& Jackson-Leach R (2007) Child overweight and obesity in the USA: prevalence rates according to IOTF definitions. Int J Pediatr Obes 2, 62-64.

25. Yngve A, De Bourdeaudhuij I, Wolf A et al. (2008) Differences in prevalence of overweight and stunting in 11-year olds across Europe: the Pro Children Study. Eur J Public Health 18, 126-130.

26. Toschke AM, Kurth BM \& von Kries R (2008) The choice of cut-offs for obesity and the effect of those values on risk factor estimation. Am J Clin Nutr 87, 292-294.

27. Wang Y (2004) Epidemiology of childhood obesity methodological aspects and guidelines: what is new? Int $J$ Obes 28, S21-S28.

28. Sweeting HN (2008) Gendered dimensions of obesity in childhood and adolescence. Nutr J 7, 1; doi:10.1186/14752891-7-1.

29. Livingstone MBE (2001) Childhood obesity in Europe: a growing concern. Public Health Nutr 4, 109-116.

30. Moreno LA, Pineda I, Rodriguez G et al. (2002) Waist circumference for the screening of the metabolic syndrome. Acta Paediatr 91, 1307-1312.

31. Freedman DS, Serdula MK, Srinivasan SR et al. (1999) Relation of circumferences and skinfold thicknesses to lipid and insulin concentrations in children and adolescents: the Bogalusa Heart Study. Am J Clin Nutr 69, 308-317.

32. Strauss RS \& Knight J (1999) Influence of the home environment on the development of obesity in children. Pediatrics 103, e85-e92.

33. Ekelund U, Neovius M, Linné Y et al. (2005) Associations between physical activity and fat mass in adolescents: the Stockholm Weight Development Study. Am J Clin Nutr 81, 355-360. 
Appendix

Public primary schools of Aveiro and children enrolled at 2nd and 3rd grade and evaluated per school

\begin{tabular}{|c|c|c|c|c|c|c|c|}
\hline Schools & Population (n) & Sample $(n)$ & Prob. (\%) & Schools & Population (n) & Sample $(n)$ & Prob. (\%) \\
\hline Group I & & & & Group IV (cont.) & & & \\
\hline School 1 & 90 & 81 & $5 \cdot 0$ & School 21 & 4 & 0 & $0 \cdot 2$ \\
\hline School 2 & 164 & 0 & $9 \cdot 1$ & School 22 & 15 & 0 & $0 \cdot 8$ \\
\hline School 3 & 40 & 0 & $2 \cdot 2$ & School 23 & 6 & 0 & 0.3 \\
\hline School 4 & 29 & 0 & $1 \cdot 6$ & School 24 & 9 & 0 & 0.5 \\
\hline School 5 & 183 & 168 & $10 \cdot 2$ & School 25 & 9 & 0 & 0.5 \\
\hline Group II & & & & Group V & & & \\
\hline School 6 & 120 & 119 & $6 \cdot 7$ & School 26 & 26 & 0 & $1 \cdot 4$ \\
\hline School 7 & 56 & 47 & $3 \cdot 1$ & School 27 & 18 & 0 & $1 \cdot 0$ \\
\hline School 8 & 37 & 0 & $2 \cdot 1$ & School 28 & 37 & 0 & $2 \cdot 1$ \\
\hline School 9 & 39 & 0 & $2 \cdot 2$ & School 29 & 12 & 0 & 0.7 \\
\hline School 10 & 23 & 21 & $1 \cdot 3$ & School 30 & 31 & 20 & $1 \cdot 7$ \\
\hline School 11 & 63 & 61 & $3 \cdot 5$ & School 31 & 64 & 47 & $3 \cdot 6$ \\
\hline Group III & & & & Group VI & & & \\
\hline School 12 & 52 & 70 & $2 \cdot 9$ & School 32 & 22 & 21 & $1 \cdot 2$ \\
\hline School 13 & 25 & 0 & $1 \cdot 4$ & School 33 & 13 & 0 & 0.7 \\
\hline School 14 & 34 & 0 & $1 \cdot 9$ & School 34 & 36 & 0 & $2 \cdot 0$ \\
\hline School 15 & 17 & 0 & 0.9 & School 35 & 28 & 0 & $1 \cdot 6$ \\
\hline School 16 & 9 & 0 & 0.5 & School 36 & 22 & 0 & $1 \cdot 2$ \\
\hline School 17 & 36 & 0 & $2 \cdot 0$ & School 37 & 17 & 17 & 0.9 \\
\hline School 18 & 14 & 0 & 0.8 & Group VII & & & \\
\hline Group IV & & & & School 38 & 40 & 0 & $2 \cdot 2$ \\
\hline School 19 & 58 & 0 & $3 \cdot 2$ & School 39 & 200 & $206^{\star}$ & $1 \overline{1} \cdot \overline{1}$ \\
\hline \multirow[t]{2}{*}{ School 20} & 60 & 53 & $3 \cdot 3$ & School 40 & 41 & 0 & $2 \cdot 3$ \\
\hline & & & \multicolumn{2}{|c|}{ Total $(I+I I+I I I+I V+V+V I+V I I)$} & 1799 & 931 & 100 \\
\hline
\end{tabular}

Population = students from 2nd and 3rd grade (aged 7-9 years); Prob. = probability to be selected (\%).

*School 39 had more students enrolled at the time of evaluation than when information was obtained. 